\title{
Nuevas tendencias locacionales del sector bancario en Madrid: la ciudad financiera del Banco Santander en Boadilla del Monte
}

\section{New local trends in the banking sector in Madrid: the financial city of the Banco Santander in Boadilla del Monte}

\author{
Eva María Martín Roda \\ Doctora en Geografía. Departamento de Geogafía. Facultad de Geografía e Historia. Universidad Nacional de Educación a Distancia (UNED). \\ Paseo Senda del Rey, 7. Edificio de Humanidades, $4^{\circ}$ y $5^{\circ}$ planta, (28040) Madrid, España, emartin@geo.uned.es \\ Silvana Sassano Luiz \\ Doctora en Geografía. Departamento de Geogafía. Facultad de Geografía e Historia. Universidad Nacional de Educación a Distancia (UNED). \\ Paseo Senda del Rey, 7. Edificio de Humanidades, $4^{\circ}$ y $5^{\circ}$ planta, (28040) Madrid, España, sfsassano@madrid.uned.es
}

Recibido: 26 de septiembre 2019 || Aprobado: 24 de abril 2020

Resumen

Boadilla del Monte, municipio de la Corona Metropolitana Oeste de la Comunidad de Madrid (CMOM), ha sufrido varios procesos de reclasificación de la tierra, de rural a urbana, como resultado del despliegue de diferentes planes de ordenación territorial. Estos planes favorecieron un notable crecimiento demográfico en el municipio, desde la segunda mitad del siglo XX y principios del siglo $X X I$, resultado del éxodo poblacional desde el interior de Madrid hasta la Corona Metropolitana Oeste de la Comunidad de Madrid. La última reclasificación creó suelo para ubicar actividades económicas. Dicha recalificación propició la llegada de actividades financieras vinculadas al sector bancario, con la implantación en el municipio de la denominada Ciudad Financiera del Banco Santander. Esta reubicación rompe con los modelos tradicionales de ubicación de las actividades financieras en los espacios urbanos centrales. A partir de este contexto, el objetivo central de este trabajo es analizar el proceso de relocalización del Banco Santander en el Municipio de Boadilla del Monte, las motivaciones que determinaron dicho traslado y su impacto en el crecimiento urbano del Municipio. La estrategia metodológica adoptada es cualitativa, basada en un minucioso trabajo de campo, realización de entrevistas en profundidad a informantes claves y en la búsqueda y revisión exhaustiva de fuentes primarias y secundarias provenientes de organismos públicos y privados.

Palabras clave: Boadilla del Monte (Comunidad de Madrid); Ciudad Financiera del Banco Santander; Expansión urbana; Terciario avanzado

Abstract

Boadilla del Monte, Municipality of the West Metropolitan Crown of the Community of Madrid, has suffered several processes of reclassification of land, from rural to urban, due the deployment of several land management plans. These plans favoured a remarkable demographic growth, from the second half of the 20th century and the beginning of the 21st century, because of the population exodus from the interior of Madrid to the Western Madrid Metropolitan Area. The last reclassification, from rural to urban, created land to locate other economic activities. This requalification led to

Cita sugerida: Martín Roda, E.M. y Sassano Luiz, S. (2020). Nuevas tendencias locacionales del sector bancario en Madrid: la ciudad financiera del Banco Santander en Boadilla del Monte. Estudios Socioterritoriales. Revista de Geografía, (27), 050. DOI: //https://doi.org/10.37.838/unicen/est.27-050 
financial development, linked to the banking sector, with the establishment in the Municipality of the so-called Santander Group's Financial City. This relocation broke with traditional models of location of financial activities in central urban spaces. From this context, the main object of this work is to analyze the relocation process of Banco Santander in the Municipality of Boadilla del Monte, the motivations that determined this transfer and its impact on the municipality's urban growth. The work methodology adopted is qualitative, based on a meticulous fieldwork, conducting indepth interviews with key informants and the exhausted search and review of primary and secondary sources from public and private organizations.

Key words: Boadilla del Monte (Madrid); Financial City of Banco Santander; Urban expansion; Advanced tertiary 
INTRODUCCIÓN

Boadilla del Monte, es un núcleo urbano emplazado en el noroeste de Madrid, en la denominada Corona Metropolitana Oeste Madrileña (desde ahora CMOM). Posee unas cualidades de conectividad y emplazamiento ventajosos que favorecen los movimientos pendulares de la población residente y la ciudad de Madrid, como asimismo con los otros municipios de dicha corona metropolitana, lo que ha favorecido la deslocalización empresarial y poblacional desde Madrid hacia dicho territorio.

Económicamente tuvo gran impacto la construcción, en el año 2004, de la Ciudad Financiera del Banco Santander (Figura 1) en la que se alojan, entre otras funciones, la Sede Corporativa del Banco y la alta dirección corporativa; además, también trasladaron desde la capital madrileña hacia este espacio diversas actividades bancarias tales como informática, servicios legales, mercado de capitales, mercados globales, banca de empresa, crédito al consumo, pagos, entre otros.

En su entorno inmediato aparecieron empresas destinadas a cubrir ciertas demandas empresariales, dedicadas a la prestación de servicios de alta cualificación o terciario avanzado y alguna de ellas participadas por el propio Banco.

El objetivo de este trabajo es analizar el proceso de relocalización del Banco Santander en la denominada "Ciudad Financiera del Santander" en el Municipio de Boadilla del Monte, e investigar su impacto en el crecimiento del entramado funcional local boadillense, como así mismo indagar las motivaciones subyacentes que determinaron el traslado de ciertas funciones hacia ese municipio.

Figura 1. Imagen Aérea de la Sede Corporativa del Banco Santander en Boadilla del Monte

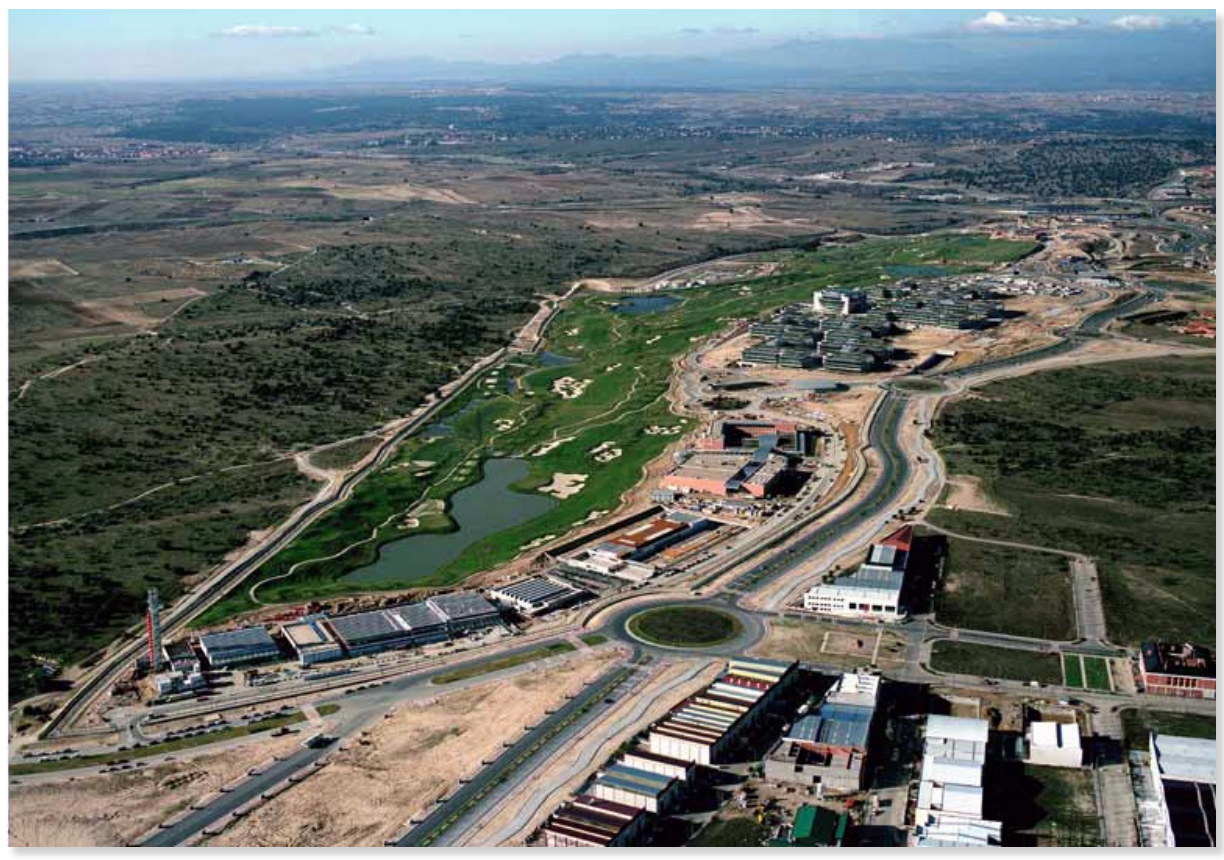

Fuente: https://www.santander.com/csgs/Satellite/CFWCSancomQP01/es_ES/Corporativo/ Acerca-del-Grupo/Sede-Corporativa/Innovaciones-en-la-construccion.html

\section{NOTAS METODOLÓGICAS}

Como fase preparatoria al estudio, se realizó una búsqueda bibliográfica exhaustiva de lo publicado en diferentes medios sobre el municipio de Boadilla del Monte. Se consultaron bibliotecas diversas, bases de datos y estadísticas, periódicos locales, publicaciones oficiales (municipales y nacionales) y prensa en general. Se realizaron diversas entrevistas al personal municipal, quie- 
nes respondieron sobre los cambios territoriales, demográficos, empresariales. Al solicitar dichos datos por escrito, se nos comunicó que, una vez consultado su servicio jurídico, no les era posible darnos tal información por escrito. No se puede obviar el hecho de que la municipalidad ha sido investigada por temas de corrupción de la denominada trama Gürtel ${ }^{1}$, lo que se considera que puede estar detrás de esta negativa.

Paralelamente se efectuó un minucioso trabajo de campo. Se realizaron varias visitas al municipio durante la investigación, lo que nos permitió familiarizarnos con la realidad espacial surgida en torno a la Ciudad Financiera, tales como nuevas empresas construidas, tipos y actividades a las que se dedican, etc. Finalmente, y para confirmar los resultados de gabinete sobre los primeros datos recabados, se realizó una última visita que sirvió para complementar las conclusiones.

La investigación espacial llevada a cabo ha tratado de entender las causas de la deslocalización de la Sede Corporativa del Banco Santander desde la centralidad de Madrid a un espacio semi-periférico, como igualmente confirmar o falsar, en palabras de Popper², la relación entre la llegada del Banco Santander a Boadilla del Monte y un posible desarrollo económico, inducido por la llegada de dicho banco.

\section{MARCO TEÓRICO}

El origen de la Geografía urbana se encuentra en Francia a principios del siglo XX; en concreto la obra de Meuriot (2018) "Aglomeraciones urbanas en Europa contemporánea", es considerada como la pionera en los estudios centrados en los procesos urbanos, aunque se pueden mencionar mu-

1 Con esta denominación se conoce una investigación policial y de la Fiscalía Anticorrupción y que está siendo juzgada ante la Audiencia Nacional; la trama está vinculada con el Partido Popular Español y se expandía principalmente por las comunidades autónomas de Madrid y Valencia.

2 Quien afirma que el conocimiento científico avanza al descartar hipótesis que contradicen la experiencia u observación. También afirma que las ciencias sociales se basan en observaciones y conjeturas más que en modelos. La ciencia debe confirmar o falsar dichas conjeturas. Popper afirmó que cualquier hipótesis, incluso en las ciencias sociales, para ser científica debe ser falsable. chos referentes como Beaujeu-Garnier y Chabot (1963), George (1961) entre otros. Si bien la ciudad siempre había sido objeto de interés de la geografía, es a partir de la segunda mitad del siglo XX cuando la ciudad y sus procesos se convirtieron en el objetivo de muchos de los estudios geográficos.

En España la producción de estudios urbanos ha sido bastante intensa a lo largo del tiempo y ha tocado numerosos temas. La producción académica ha analizado tanto aspectos funcionales como sociales o económicos, según la finalidad de la investigación. También fueron objeto de interés de la Geografía Urbana los aspectos relacionales de la ciudad con su entorno espacial a diferentes escalas: regional, nacional e incluso mundial, sin olvidarse de los procesos intraurbanos. Desde el punto de vista regional, o autonómico, muchos estudios se centraron en el análisis de los intercambios, de los flujos y de los procesos de localización y relocalización espacial, tanto poblacionales como económicos y comerciales.

Sin embargo, los estudios de los procesos de relocalización espacial en la corona metropolitana del oeste madrileño han parecido perder el interés de los investigadores en los últimos años, si bien algunas tesis doctorales sí han analizado el tema de la reestructuración económica y social en el conjunto del área metropolitana madrileña, como la tesis defendida en el año 1987 por Constanza Tobío Soler titulada "La reestructuración económico-social en el área metropolitana de Madrid 1970-1985". Un tema tratado recientemente, en referencia al área metropolitana madrileña, ha sido el poli-centrismo y el crecimiento espacial en la tesis doctoral titulada "Del área metropolitana hacia la región urbana policéntrica madrileña: cambio de escala, estructura y articulación territorial" (Solís Trapero, 2011); no obstante, el área metropolitana de Madrid y las transformaciones que en la misma tienen lugar no parecen suscitar un interés especial en el momento actual.

Es por todo ello por lo que, conscientes de los procesos internacionales de utilización del espacio urbano y metropolitano en Madrid, como 
medio financiero de extracción de plusvalías de la ciudad (Sassen, 2018), se ha planteado este estudio sobre la relocalización de la Sede corporativa del Banco Santander en Boadilla del Monte y sus posibles motivaciones: "existe una enorme maquinaria económica que impone sus reglas sobre todos los aspectos de la vida metropolitana" (Saldarriaga Roa, 2016, p.13).

\section{EL ÁREA METROPOLITANA DEL OESTE MADRILEÑO}

En este artículo, salvo indicación expresa en contra, con la definición de Corona Metropolitana Oeste Madrileña (CMOM) se hará referencia en exclusiva a los municipios que tradicionalmente tuvieron la consideración de primera corona en la zona oeste de Madrid. Estos municipios son los que inicialmente se incorporaron al proceso de metropolización: Boadilla del Monte, Majadahonda, Pozuelo de Alarcón, Las Rozas de Madrid,
Torrelodones y Villaviciosa de Odón (Figura 2); todos ellos se encuentran en el denominado tejido urbano continuo, interconectado por carreteras y autopistas nacionales o por vías de circunvalación y redes de alta velocidad. Estos espacios suelen tener una elevada concentración residencial y de actividades económicas, "existe una tendencia general a que el peso del tejido urbano continuo sea mayor conforme aumenta el índice de densidad de la red". (Salom y Albertos Puebla, 2010, p.72).

Toda la corona, incluida Boadilla del Monte, sufrió los principales cambios poblacionales, económicos y funcionales a partir de la segunda mitad del siglo XX y los primeros años del siglo XXI, como consecuencia de las transformaciones acaecidas en los diferentes planes de ordenación territorial (OT). Pese a ello, la densidad poblacional en la CMOM no es elevada, lo que confiere al territorio una elevada calidad medioambiental (Figura 3).

Figura 2. Mapa Comunidad de Madrid

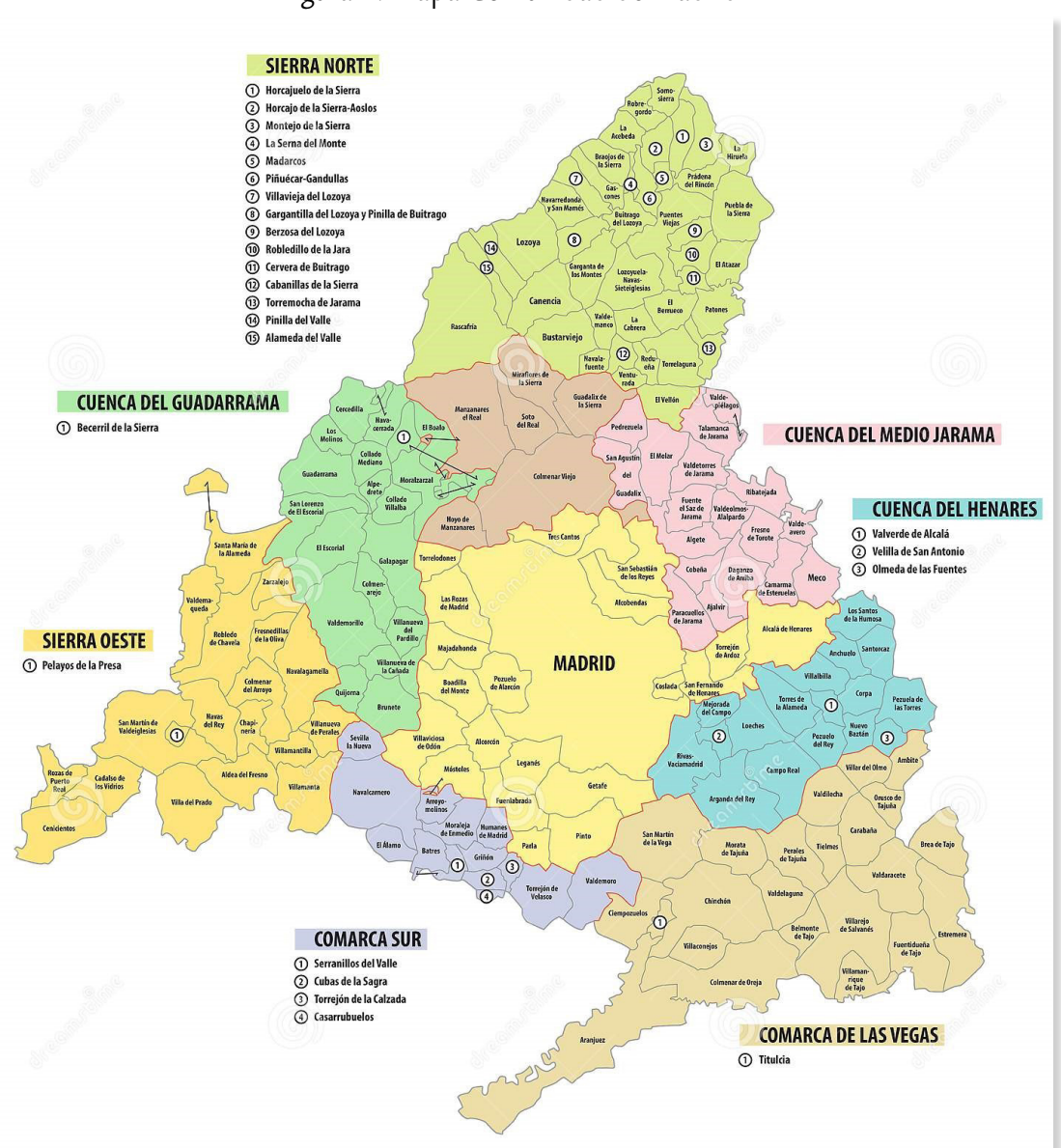

Fuente: Ilustración 129420214 @ Rainer Lesniewski: Libre De Derechos. Obtenido el 27/03/2020 https://es.dreamstime.com/ comunidad-de-mapa-administrativo-y-pol\%C3\%ADtico-madrid-del-vector-image129420214\#_Dreamstime.com 
Figura 3. Densidad poblacional de algunos municipios de la CMOM en el año 2016

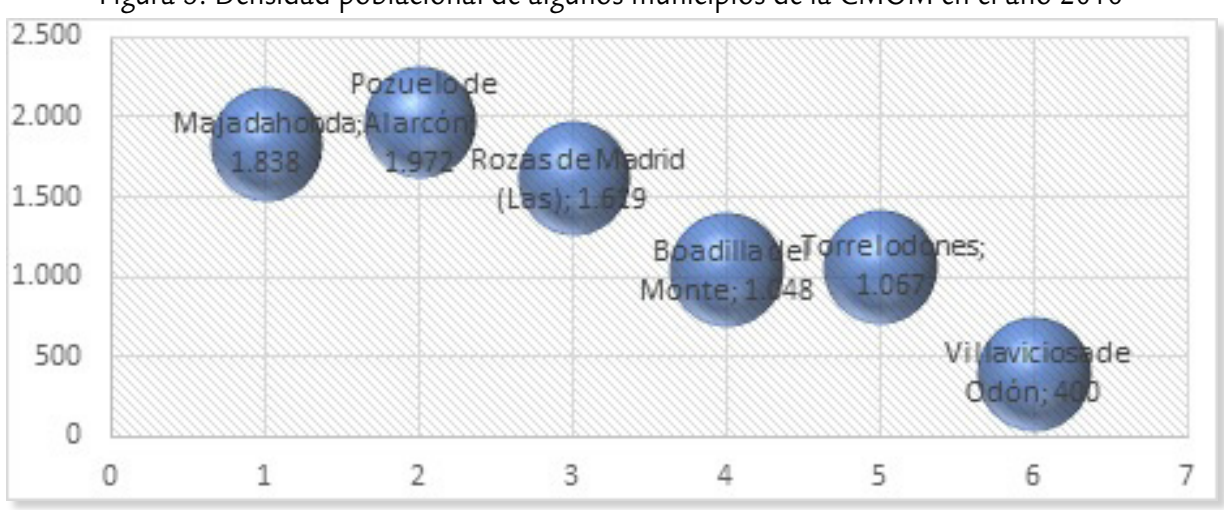

Fuente: Instituto de Estadística de la Comunidad de Madrid

En Boadilla del Monte los cambios socio espaciales más importantes derivan del Plan General de Ordenación Urbana (PGOU) del año 2001, que es el que más afectó al territorio objeto de este estudio, a pesar de que dicho plan ha estado envuelto en diferentes procesos "Contencioso Administrativo" hasta que fue anulado por el Tribunal Supremo en el año 2015. El PGOU del año 2001, que siguió adelante mientras no hubo sentencia anulatoria en firme, facilitó el crecimiento poblacional y económico del término municipal, mediante la recalificación del suelo rural a suelo urbano destinado a albergar nuevas actividades, tanto económicas como habitacionales.

Desde el punto de vista de la inclusión de Boadilla del Monte en la CMOM se desea hacer una pequeña reflexión al respecto pues, en general, clasificar territorios suele ser una tarea imprecisa en la mayoría de los casos. Para destacar la complejidad del clúster de los municipios que pueden pertenecer a la CMOM, los municipios de Majadahonda, Las Rozas de Madrid y Boadilla del Monte, que se han incluido en esta clasificación, en el Atlas Estadístico del Ministerio de Fomento tienen la consideración de áreas urbanas, lo que corrobora la dificultad encontrada a la hora de incluir en un clúster el espacio objeto de este estudio.

De la CMOM se ha excluido, al no influir en este estudio, a Villanueva de la Cañada y Villanueva del Pardillo, a pesar de que algunos estudios los incluyen en la CMOM y de que en ellos se observan interesantes procesos económicos que los están incorporando al mercado productivo, "por lo que su actividad va más allá de la exclusividad de un submercado territorial de la vivienda" (Feria Toribio, 2010, p. 36).

El modelo espacial productivo madrileño. El FIDI VERSUS EL CBD

A principios del Siglo $X X$, autores de la escuela de Chicago como Burges, Hoyt, Ullman y Harris entre otros, ya utilizaban el concepto de Central Business District (CBD) para definir el área central de una ciudad donde se concentra el transporte, actividades de negocios, servicios, comercio, empresas financieras, vehículos con buena accesibilidad y alto precio del suelo.

Por su parte, los distritos financieros o Financial District, (FiDi), “(...) nacen en un intento de concentrar geográficamente las actividades financieras, al igual que los gremios se agrupaban por oficios en una misma calle o barrio en la época medieval" (Pérez Mejías, 2012, p. 2). Los mismos se caracterizan por tener un paisaje peculiar de grandes torres que se asemejan en algunos aspectos a las características propias del denominado distrito de negocios (CBD), aunque difieren en ciertos aspectos como, por ejemplo, en las actividades muy concretas que en el mismo se desarrollan. El FiDi, en función del rango de la ciudad en el que se localice ha tenido, y tiene, diferentes delimitaciones y estructuras. En realidad, a lo largo del tiempo el FiDi ha evolucionado incluso desde el punto de vista arquitectónico, desde edificios con aspecto de 
solidez y construidos con materiales considerados nobles, como el granito o el mármol, que trataban de generar una imagen de solvencia económica, hasta construcciones más modernas y ligeras ligadas a la estructura del cristal, que configuran auténticos edificios pantalla.

Habitualmente, en las grandes ciudades globales como Nueva York o Londres, el FiDi se emplaza en lugares centrales. Para incrementar su funcionalidad y favorecer el rápido acceso al mismo se sitúa en espacios de gran actividad comercial y económica, lo que confiere al suelo elevados precios. A veces estos distritos financieros están integrados en un CBD, pero en otras no y conforman agrupaciones independientes, en función de su tamaño. Si se piensa en el centro financiero mundial por excelencia, la ciudad de Nueva York, se aprecia claramente la diferencia entre ambos espacios: el financiero $y$ el de negocios. El FiDi contiene en exclusiva las oficinas y algunas sedes de muchas de las principales instituciones financieras de la ciudad y del mundo, como la Bolsa de Nueva York, el Banco de la Reserva Federal de Nueva York, la New York Mercantile Exchange, el NASDAQ, el New York Board of Trade, las sedes sociales de grandes fondos de inversión institucionales y privados; y las mayores sedes sociales bancarias, muchas de ellas ubicadas en la conocida Wall Street, corazón del FiDi neoyorquino.

Las diferencias entre ambos espacios, CBD y FiDi son, en algunos casos, sutiles y sus disparidades van a depender, en gran medida, de la jerarquía económica global de la ciudad en la que se integren. En las ciudades globales de categoría alfa, como Nueva York o Londres, el CBD y el FiDi suelen estar diferenciados. En las ciudades de menor rango global, ambos distritos suelen estar integrados y forman un único conjunto.

En el caso particular del espacio urbano madrileño, su entramado productivo y residencial puede ser definido como espacio de convivencia. En general, en el interior de Madrid, la localización de las actividades económicas responde más a un modelo disperso y de convivencia que a un modelo de alta concentración en un CBD.
Las actividades económicas se entremezclan con los espacios residenciales, salvo algunas excepciones, como el denominado Centro AZCA o el parque empresarial denominado Cuatro Torres Business Área (CTBA). En los espacios de borde las actividades productivas se sitúan junto a las vías de alta velocidad como la M-30, M-40 o carreteras nacionales, muchas veces realizan un efecto pantalla para las zonas residenciales que se localizan detrás de estas áreas destinadas al terciario y a veces al sector industrial denominado limpio. Esta mixtura de actividades económicas y residenciales, si bien hace que en Madrid no se pueda hablar propiamente de un CBD o de un FiDi, evita los efectos que la segregación funcional provoca y dinamizan económicamente el entorno inmediato en el que se establecen, al atraer aquellos servicios demandados por empleadores y empleados, como agencias bancarias, restaurantes, gimnasios, cafeterías, dinamización que no se produce en el caso de la deslocalización llevada a cabo por el Banco Santander en Boadilla del Monte.

En Madrid no ha existido como tal, algo que pueda ser definido como un FiDi, si bien, históricamente las sedes sociales de los principales bancos españoles se emplazaban en la zona central del espacio urbano madrileño, que describían un área triangular denominada por José Sanz García (1975) como triángulo del capital español y que estaba definido por los vértices de Puerta del Sol-plaza de Cibeles-plaza de la Lealtad. En la actualidad en este espacio, salvo el Banco de España y la Bolsa de Valores, no existe ninguna sede de los grandes bancos españoles ni de grandes empresas financieras. Su funcionalidad evolucionó hacia la actividad principalmente comercial y hostelera, vinculada al desarrollo turístico.

Algunos de los municipios madrileños, a pesar de tener un carácter intermedio y como consecuencia de los procesos de deslocalización y globalización productiva, pueden llegar a desempeñar funciones importantes en la organización territorial y en las actividades económicas del entorno urbano al que pertenecen, 
al favorecer el desarrollo o la consolidación de mega-regiones (Sassen, 2007). Este es el caso de Boadilla del Monte que, de ser un municipio fundamentalmente residencial y de baja densidad poblacional dentro de la CMOM (Figura 3), se ha convertido en un importante centro económico ligado al sector financiero y de servicios empresariales avanzados, sobre todo en la última década del siglo XX y primera del siglo XXI gracias a la deslocalización, fundamentalmente de las funciones corporativas, llevada a cabo en el año 2004 por el grupo del Banco Santander mediante la construcción de la Ciudad Financiera del Banco Santander.

El banco comienza sus actividades en 1857 en Santander, ligado al comercio con Iberoamérica. En 1923 desarrollan una red de sucursales en el norte del país. Desde 1950 comienza un gran período de expansión con la adquisición de numerosos bancos locales y apertura de oficinas en varios países latinoamericanos, situación que continúa en la actualidad. Alrededor de 200.000 profesionales trabajan en todo el mundo para este banco.

\section{DISPERSIÓN ESPACIAL DE LAS SEDES SOCIALES BANCARIAS ESPAÑOLAS}

Desde los años ochenta del siglo XX y fundamentalmente después de la crisis económica del año 2008, se asistió en Madrid a una primera deslocalización de las sedes sociales de los grandes bancos españoles, desde el triángulo del capital español, hacia otros distritos centrales. En dicho triángulo, ha tenido lugar un proceso de sustitución funcional, cuyo proyecto más emblemático es el que se sitúa en el espacio en el que se alojaban las sedes del Banco Central Hispano y la del Banco Español de Crédito, cerca de la Puerta del Sol. Ambos bancos fueron adquiridos por el Banco Santander. Los edificios, conocidos en Madrid como "Complejo Canalejas", fueron vendidos a una constructora en el año 2014 por 215 millones de euros. En esta operación se hizo explicito lo que Saskia Sassen denomina como "extracción de plusvalía de la ciudad” (Rodriguez-Rata, 2018). La operación le supuso al Banco Santander una plusvalía de 85 millones de euros, según informó en su momento la Comisión Nacional del Mercado de Valores. En dicho complejo se está construyendo en la actualidad un conjunto comercial y hotelero de lujo; el primero contará con 16.000 metros cuadrados de tiendas, el segundo será un hotel de cinco estrellas de la lujosa cadena Four Seasons, el proyecto también contempla la construcción de 22 viviendas de lujo.

A partir del año 2000 la dispersión nacional del sector financiero ha sido aún más evidente. En ese primer proceso de deslocalización, la autora Martín Roda afirma que: "el triángulo de negocios (...) se ha desplazado hacia el norte, a lo largo del eje Prado-Castellana, CBD. (...) como el anteriormente citado Santander-BBVA o el británico Barclays Bank, situado en la plaza de Colón, en las antiguas Torres del mismo nombre que pertenecieron al expropiado grupo español Rumasa” (Martín Roda, 2000, 236).

Esta dispersión espacial tiene su origen en la división territorial en comunidades ya que la mayoría de las entidades financieras autonómicas, y de implantación exclusivamente nacional, mantienen las sedes sociales en sus propias autonomías sin valorar la cercanía a los centros de decisión nacional ni tampoco los beneficios derivados de la economía de escala. Esto es por motivos meramente económicos y fiscales, pues es en aquellos territorios donde se pagan los impuestos e influyen en las cifras totales del Valor Agregado Bruto autonómico ${ }^{3}$.

En la actualidad, mantienen sus sedes sociales en Madrid Bankinter y Liber-Bank y tan solo los dos grandes bancos internacionalizados, y con proyección global: BBVA y Santander. Bankinter, un banco de tamaño medio, dedicado a banca comercial y a banca de empresas, mantiene su sede en el Paseo de la Castellana; Liber-Bank, banco cuyo origen procede de la fusión de diversas cajas de ahorro, se ha emplazado entre las

3 Valor Agregado Bruto autonómico es un indicador macroeconómico que nos dice la producción total de bienes y servicios en un ámbito territorial concreto, en este caso, en cada Comunidad Autónoma. 
autopistas $\mathrm{M}-11$ y la A-1, en el barrio denominado Virgen del Cortijo, lejos del espacio central y más representativo madrileño. El BBVA ha construido un nuevo complejo corporativo en un espacio de menor centralidad, en el barrio de Las Tablas, en la zona norte de Madrid y, el Banco Santander, como ya se ha indicado, en Boadilla del Monte, en la CMOM. Según Forbes el Banco Santander, Telefónica, el BBVA, Iberdrola, Gas Natural e Inditex constituyen las empresas globales españolas más emblemáticas internacionalmente (Mazo, 2016).

Como ha señalado recientemente la Organización para la Cooperación y Desarrollo Económicos (OCDE), en el año 2003, el Banco Bilbao Vizcaya Argentaria (BBVA) y el Banco Santander Central Hispano (BSCH) "se situaban en los dos primeros puestos del ranking de los mayores bancos en Latinoamérica por activos consolidados (62.894 y 61.019 millones USD respectivamente), según datos de la Comisión Económica para América Latina y Caribe-CEPAL (OCDE, 2007)". (Sánchez Moral, Calatrava Andrés y Melero Guilló, 2008, p. 32).

En España, la nueva realidad locacional de las funciones financieras, difieren de las peculiaridades de concentración y centralidad que eran hasta ahora comúnmente aceptadas. La marcha del Banco Santander del espacio central urbano madrileño hacia el espacio metropolitano es un modelo diferenciado de radicación. En realidad, en Madrid se está produciendo una transformación peculiar que se caracteriza por la dispersión territorial nacional de las sedes sociales y corporativas bancarias, revolucionando el comúnmente aceptado modelo de concentración espacial central de las actividades financieras. Existe por tanto, un proceso de deslocalización y dispersión espacial a escala nacional de las sedes sociales bancarias de las entidades españolas, siendo un modelo peculiar español.

INTEGRACIÓN FINANCIERA DEL BANCO SANTANDER Y BBVA

El Banco Santander, antes de la crisis, había realizado la compra e integración jurídica de diferentes entidades como la del banco Hispano Americano, que a su vez había absorbido al banco Central, ambos con sede social en Madrid antes de ser integrados. El BBVA por su parte sumó a Argentaria, grupo del que formaban parte el banco Hipotecario y el banco Exterior de España, cuyas sedes sociales estaban radicadas en Madrid. Como consecuencia de estas integraciones, en el año 2006, los denominados siete grandes bancos españoles: Hispano Americano, Español de Crédito (Banesto), Popular Español, Bilbao, Vizcaya - Central y Santander, tan solo mantenían la sede social en Madrid los bancos Popular Español, emplazado en el emblemático Edificio Beatriz, en la calle Ortega y Gasset, y Banesto cuya sede social se encontraba en la plaza de Canalejas. Madrid ya no podía ser definida como la Sede del Capital Español.

Después de la crisis de 2008, protagonizada principalmente por las cajas de ahorro y cuyas sedes se localizaban en las capitales de las diferentes autonomías, se reorganiza prácticamente todo el sector bancario español. Son numerosas las integraciones que se producen, entre ellas la de Banesto, perteneciente jurídicamente al Banco Santander desde el año 2013 y la del Banco Popular Español al que compró en el año 2017, por la simbólica cantidad de $1 €$, a lo que se debe sumar toda la deuda y obligaciones acumuladas por el Banco Popular.

Por su parte el Banco Popular Español, antes de ser absorbido por el Banco Santander, también había iniciado un proceso de relocalización desde espacios centrales a distritos periféricos (barrios de Hortaleza y San Blas), cerca del Campo de las Naciones, donde la renta de situación es menor ${ }^{4}$. Esta absorción le ha permitido al banco Santander realizar una importante operación inmobiliaria con sus oficinas ubicadas en el denominado complejo Banesto -Mesena. El proceso se inició con el traslado de los empleados que estaban en la calle Mesena,

4 Más de $5.000 €$ el m2 construido frente a los $2.500 € \mathrm{el} \mathrm{m} 2$ construido en su nueva localización (Fuente: https://www. idealista.com/. Revista de compra venta de inmuebles. Precio de referencia al realizar el artículo) 
a los edificios construidos por el Banco Popular Español en la autopista A-II. Seguidamente se inició la tramitación de la venta del complejo Banesto - Mesena, aproximadamente 164.000 $\mathrm{m} 2$ de terrenos, cuya calificación urbanística es tanto terciaria como residencial. Se trata de un espacio de gran valor económico al encontrarse cerca de la calle Arturo Soria, una de las zonas más caras y valoradas de Madrid 5 .

Para el banco se trata de otra operación de extracción de plusvalías urbanas en un momento en el que las actividades bancarias tienen menores márgenes empresariales, como consecuencia del bajo tipo de interés de intervención permitido por el Banco Central Español, del aumento del capital regulatorio y del descenso de la concesión de créditos e hipotecas. Por ello los bancos y fondos de inversión, como medio para incrementar rentabilidades diversifican sus actividades dirigiendo sus inversiones hacia otros sectores, como por ejemplo adquisiciones de participaciones en Socimis, caso de Bankinter. En otros casos como el del Banco Santander, que cuenta con numerosos activos inmovilizados en el sector inmobiliario, incrementa sus beneficios mediante desinversiones de la cartera de los activos en inmuebles. La compraventa del complejo Banesto - Mesena se considera una de las mayores operaciones inmobiliarias de activos terciarios y residenciales que han tenido lugar en Madrid en el 2019, pero no se consiguió información del monto de la operación.

En Madrid, por tanto, existe un proceso peculiar y diferenciado de dispersión de las sedes sociales bancarias, tanto en el espacio interior urbano madrileño, como por todo el territorio nacional, lo que da al traste en Madrid con el tradicional modelo de concentración espacial de las sedes financieras y ban-

5 El precio de suelo urbano en el interior de Madrid según Certicalia (https://www.certicalia.com/precio/valoracion-suelo-urbano/madrid/madrid/madrid) oscila entre $1.581 €$ el m2 y $1.950 € \mathrm{el}$ $\mathrm{m} 2$; en un municipio de borde, como San Sebastián de los Reyes, Acciona ha pagado $1.500 €$ el m2 (https://hortanordgie.es) carias, que había sido descrito por José Sanz García para el espacio capitalino.

\section{El Banco Santander: deslocalización hacia Boadilla DEL MONTE}

Nuestro caso paradigmático de estudio es la Ciudad Financiera del Banco Santander, que no se ha marchado desde el espacio central urbano hacia un distrito periférico, sino que se estableció en el espacio metropolitano del oeste de la capital, modelo de radicación diferenciado del resto de procesos de relocalización de otras entidades bancarias y cuya causalidad es el objeto de este trabajo (Figura 4).

El proceso de relocalización de las sedes corporativas bancarias emplazadas en Madrid, hacia espacios metropolitanos, como ha hecho el Banco Santander, no ha sido secundado por otros bancos, lo que denota la preferencia de las entidades financieras madrileñas por espacios urbanos de borde, como el caso ya mencionado del BBVA, y no periféricos.

La Ciudad Financiera del Banco Santander en Boadilla del Monte ocupaba, inicialmente, una superficie de 165 hectáreas, superficie que se vio ampliada con posterioridad a 250 hectáreas. El complejo está compuesto por siete edificios destinados a oficinas, entre los que se encuentra el Edificio Pereda, que aloja el Consejo y la alta dirección; está planificado como una ciudad autónoma diseñada para alojar a más de 6.000 empleados "La ciudad en la que trabajan 6.700 empleados, recibe mensualmente unos 15.000 visitantes, en su mayoría clientes y proveedores procedentes de todo el mundo" (Jodidio, 2012, p. 115).

Es un espacio encerrado en sí mismo y aislado de su entorno inmediato. El perímetro está rodeado de muros y vallas, con accesos vigilados por guardias de seguridad que controlan la entrada y salida de empleados y visitantes. Aquellos empleados que llegan mediante el transporte público disponen en su interior de autobuses que recorren el recinto de un edificio a otro. 
Figura 4. Principales áreas funcionales y principales corredores funcionales

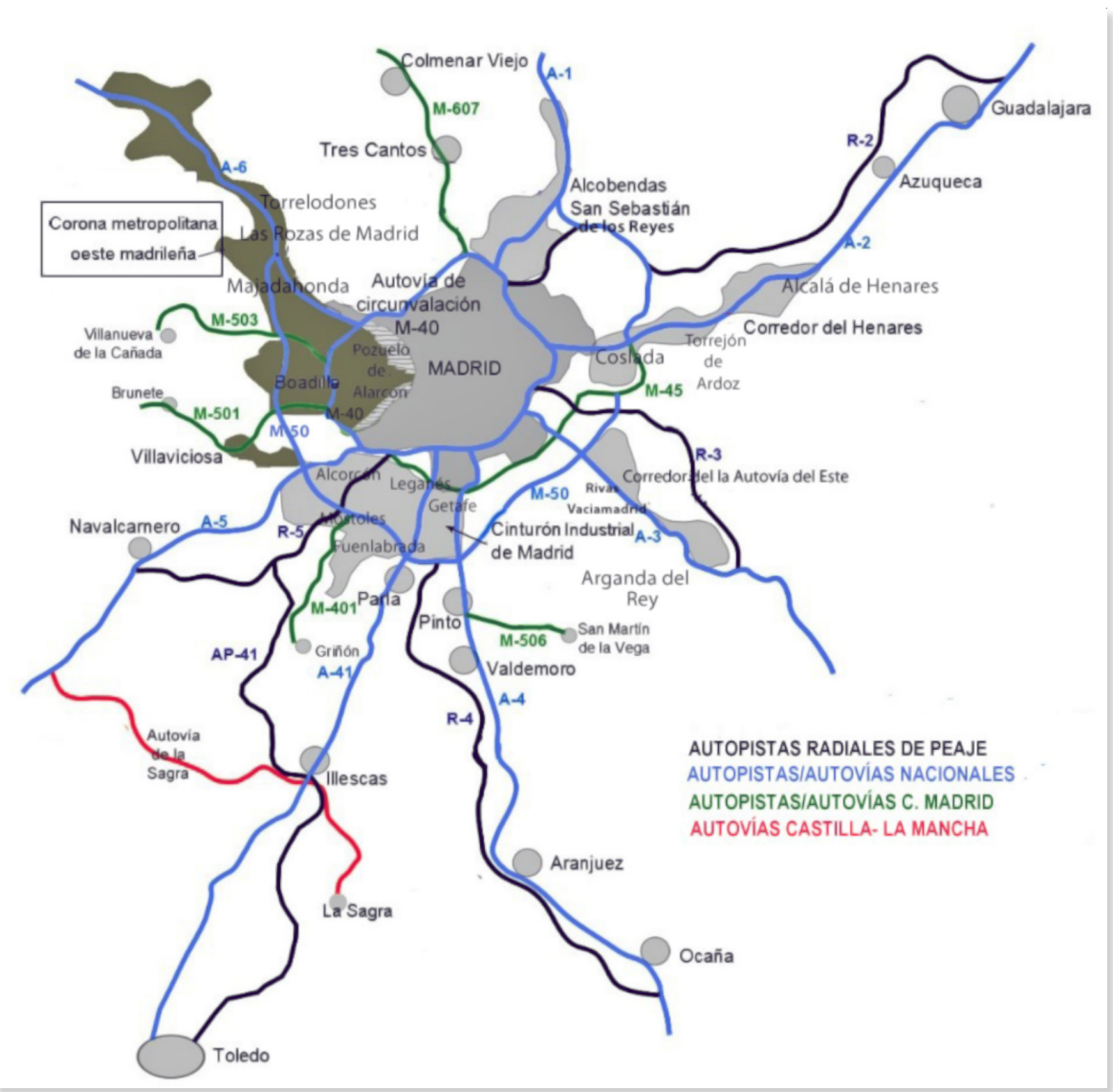

Fuente: https://sextacolumna.wordpress.com/2012/11/12/relato-de-la-fiesta-ii/

Todo el complejo posee una alta calidad medioambiental. Está emplazado en medio de jardines, con especies florales de alto valor ecológico, como los olivos centenarios que lo adornan y que fueron llevados allí exprofeso. Posee incluso, un campo de Golf. En este entorno natural privilegiado se concentran los edificios corporativos que albergan a la dirección y a los empleados, junto a numerosos y diversos servicios, tales como restaurantes, tiendas, tintorería, clínica, peluquería, gimnasio con piscina climatizada, guardería (de uso exclusivo para los empleados que tienen su puesto de trabajo en el recinto), hotel, sala multiusos (conferencias/teatro/convenciones), aparcamientos de uso limitado a los empleados del recinto e invitados, lo que hace del lugar un espacio autosuficiente frente a las demandas de servicios personales que pudiesen tener sus empleados (Torquemada, 2012).

La conectividad con el centro urbano de Ma- drid mediante transporte público es dificultosa (Figura 4). A las inmediaciones de la Ciudad Financiera del Banco Santander, en Boadilla del Monte, tan solo llegan, de forma directa las líneas de autobuses metropolitanos 574, que nace en Aluche, y la línea 3 del denominado metro ligero (ML), que empieza en la Ciudad Jardín, emplazada en el barrio de Carabanchel, en el distrito de la Latina. La línea 3 del ML, en su cabecera, enlaza con la línea 2 del también MLy con la línea 10 del metropolitano madrileño. El Banco, no obstante, tiene a disposición de sus empleados lanzaderas desde diferentes puntos de Madrid (Moncloa, Aluche y Avenida de América), cuya frecuencia y horario varían en función del lugar de origen y de la estación del año. En general, este centro empresarial es un espacio periférico de fácil y rápido acceso por automóvil, aunque no tanto mediante el transporte público, por ello se puede hablar de una descentrali- 
zación jerarquizada, semejante a la definida en el año 1990 por Herbert y Thomas (1990).

Estas peculiaridades de funcionamiento han permitido que no se produzcan distorsiones en las características sociales del municipio, pero tampoco han inducido un incremento de las actividades económicas locales al no generarse las sinergias económicas positivas consecuencia del incremento en la demanda de servicios personales tales como restauración, hostelería, servicios empresariales, pues la Ciudad Financiera es autosuficiente en este aspecto. Tampoco se ha ocasionado la radicación de proveedores de servicios empresariales en su entorno cercano pues, las compañías de servicios avanzados que han aparecido junto a la Ciudad Financiera son empresas cuyo capital mayoritario pertenece o está participado por el propio banco. El principal efecto que se ha producido en el entorno de la Ciudad Financiera ha sido la presión ejercida sobre la red viaria, causada por los movimientos pendulares diarios de los aproximadamente 6.000 empleados que allí trabajan, como por los visitantes que a diario son recibidos en el complejo.

Este centro empresarial es un modelo urbanístico pionero y novedoso en Madrid por su carácter cerrado, que contrasta con otras grandes actuaciones urbanísticas de complejos empresariales de carácter abierto llevadas a cabo en el entorno urbano madrileño, como la Sede de Telefónica. No obstante, estos centros empresariales aislados son habituales en otros entornos geográficos, como EE.UU., y comunes a otras actividades como las de alta tecnología de empresas como Apple, IBM, por citar algunos ejemplos.

\section{SINGULARIDADES DEL CONJUNTO ARQUITECTÓNICO}

La Ciudad Financiera fue diseñada por el arquitecto Kevin Roche que, en una importante apuesta por mantener la calidad medioambiental del entorno (cercanía del Parque Regional de Guadarrama) y la sostenibilidad del conjunto, cubrió los tejados de todos los edificios de vegetación lo que proporciona al edificio aislamiento térmico y acústico, de manera natural y ecológica.
El paisaje del conjunto parece orientado a ocasionar una sensación de libertad para las personas que diariamente lo habitan. (...) la arquitectura (...) definida por formas cerradas, (...) por el uso de formas delimitadas, (...) produce una sensación de plenitud, de satisfacción (...) la arquitectura de formas abiertas es la que, sin prescindir totalmente del orden, quiere dar apariencia de libertad. (Perelló Ferrer, 1991, p. 40-41)

El conjunto en el año 2013 recibió el premio The Work-Life Innovative Excellence Award a la excelencia en conciliación, concedido anualmente por Alliance for Work-Life Progress, organización norteamericana que premia a aquellas empresas que impulsan entre sus empleados la conciliación entre desarrollo profesional y su vida personal. Ha sido la primera vez que una empresa europea consigue este galardón. La Ciudad Financiera tiene una estructura arquitectónica más propia de las empresas de tecnología de punta que la habitual, hasta el momento, de los edificios bancarios. Su modelo arquitectónico responde al estándar de sedes empresariales como la de IBM en Armonk (Nueva York), Microsoft en Redmond (Washington) o la nueva sede de Apple en Cupertino (California), en las que la protección de la tecnología y de las investigaciones que en las mismas se realizan son la principal prioridad, de ahí su aislamiento y la limitación de la entrada al público en general, aislamiento que hasta ahora no era propio del sector bancario. Tradicionalmente, los grandes bancos perseguían, a través de sus edificios, dar sensación de solidez, mediante el uso de materiales nobles en sus fachadas tales como el granito o el mármol, a la par que aportar una imagen de transparencia por lo que sus puertas suelen estar a pie de calle y abiertas al público en general; en este complejo no se ha optado por seguir con la tradición constructiva habitual y propia de las entidades financieras.

Sin embargo, el conjunto sí que está diseñado para impactar a las personas que acceden al mismo, al reflejar sus novedosos diseños el estatus y la importancia internacional de la institución bancaria. "El hierro finamente forjado y el cristal que parece desafiar la gravedad pue- 
den contener los mismos mensajes que el robusto mármol o el bronce y definir igual de bien las instituciones consideradas por la sociedad como las más importantes o más significativas" (Sudjic, 2009, p. 37). En el diseño realizado por Roche, en el que se entremezcla lo financiero con lo arquitectónico y con un modo de trabajo que busca el bienestar de sus empleados, se advierte el interés por construir una marca atractiva y diferenciada para la Ciudad Financiera y de distinción para la imagen proyectada por el banco.

\section{¿Por Qué Boadilla del Monte?}

Una de las preguntas que nos planteamos al comienzo de este trabajo, fue por qué el Banco Santander elige Boadilla del Monte y no un espacio en el interior urbano, tal y como hicieron Telefónica o el BBVA.

Sin lugar a dudas el factor de conectividad de Boadilla del Monte con la capital de Madrid debió de ser un elemento importante de decisión, pues las nuevas lógicas de localización están directamente relacionadas con la velocidad de conexión a la red viaria. Los terrenos en los que se ubicó la Ciudad Financiera están cerca de Madrid (14,5 km), emplazados en la corona metropolitana oeste, bien comunicados (entre las autovías M-50, M-40 y la M-501) (Figura 5).

El municipio se encuentra rodeado por los de Villanueva de la Cañada, Majadahonda, Pozuelo de Alarcón y Villaviciosa de Odón. Los tres primeros están entre los 20 municipios de España con renta per cápita más elevada, ocupando Boadilla del Monte el segundo lugar en el ranking. El territorio tiene también un elevado valor medioambiental (Boadilla del Monte tiene 793 hectáreas en el denominado Parque Regional del Curso Medio del río Guadarrama y amplios espacios boscosos protegidos) (Arnaiz, 2007; Ayuntamiento de Boadilla del Monte, 2003; Época, 2003).

Sin embargo, dos de los componentes que se consideran importantes en la radicación empresarial, como son la cercanía aeroportuaria y la economía de escala, son factores que no se corresponden con la localización de la Ciudad
Financiera. Es conocido que para la construcción de esta se barajó la posibilidad de situarla en el municipio de Alcobendas, donde era posible adquirir terrenos junto a la localidad de la Moraleja, barrio con la mayor renta per cápita de España. Desde el punto de vista de conectividad, Alcobendas, está mucho mejor emplazada y conectada que Boadilla del Monte pues, en su entorno, se encuentran importantes vías de comunicación como son la M-30, M-40, A-1, R-2, M-50, M-603 y también al aeropuerto de Madrid-Barajas-Adolfo Suárez. Además, Alcobendas también está mucho más cercana al eje del Paseo de la Castellana, importante vía vertebradora de las comunicaciones interiores en la ciudad de Madrid de lo que lo está Boadilla del Monte; $y$ en Alcobendas se encuentran numerosas empresas internacionales, muchas de ellas proveedoras de servicios empresariales (Martín Roda, 2000), lo que hace difícil entender, solo por factores de conectividad, la decisión sobre la localización de la Ciudad Financiera en el espacio metropolitano oeste de Madrid.

Aunque en las decisiones finales de radicación siempre existe un determinado grado de subjetividad "Los hechos geográficos deben ser estudiados (...) con arreglo a los tres clásicos y conocidos principios de localización, explicación y extensión" (Sanz García, 1975, p. 17). En este caso, y después de analizar todo el material recopilado, se estima que el factor concluyente de elección radica en una compleja operación de obtención de plusvalías ${ }^{6}$, como explica detalladamente en su artículo Álvaro Ardúa Urquiaga (2014), mediante la recalificación de suelo declarado como rústico a suelo de uso terciario y residencial. En los suelos recalificados como residenciales se planificó también la construcción de 3.000 nuevas viviendas ${ }^{7}$, base del reciente crecimiento poblacional del municipio.

6 Para determinar este factor se contactó con Arnaiz Consultores, gestores en el desarrollo planificador de la Ciudad Financiera y del desarrollo del último POT de Boadilla del Monte, pero declinaron ofrecernos la información sobre cuáles fueron los factores decisionales.

7 Fuente: http://www.nodo50.org/derechosparatodos/DerechosRevista/Derechos16-Ladrillos.htm 
Figura 5. M- 501: frontera entre la zona residencial y la zona industrial y de servicios

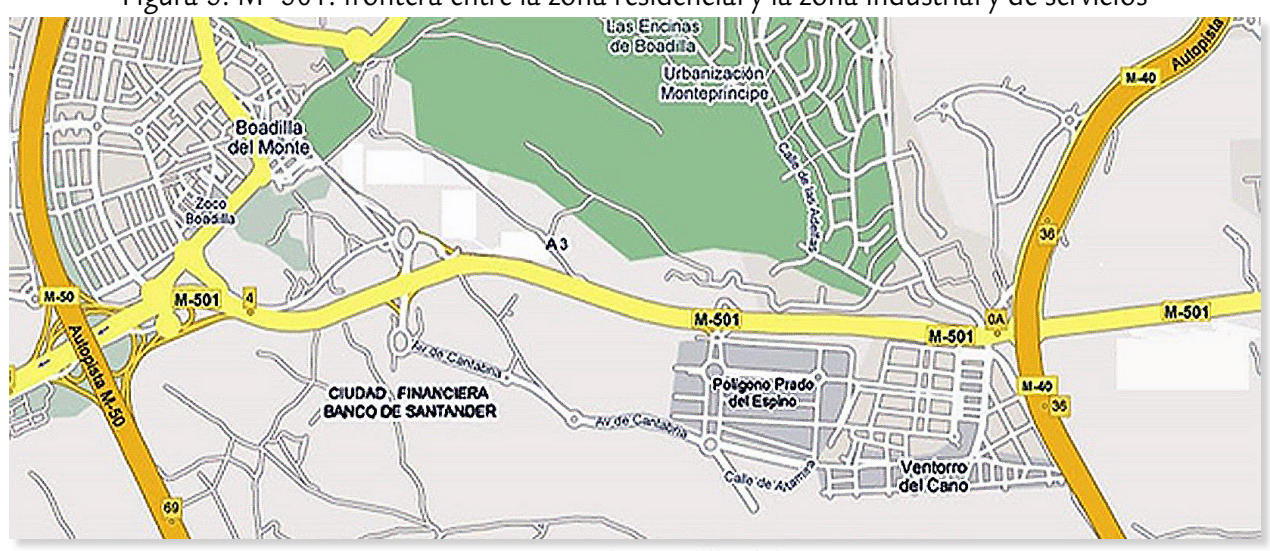

Fuente: Ayuntamiento de Boadilla del Monte, 2017

La CIUDAd FINANCIERA DEl Banco Santander en Boadilla del Monte: Plusvalías GeNeradas

El coste de construcción de la Ciudad Financiera fue elevado, aunque no tanto como podría haber sido su construcción en el interior de la ciudad de Madrid, incluso en los distritos de borde o periféricos, sobre todo por el diferencial en el coste del suelo calificado como urbano y el suelo calificado como rural. Se estima que la construcción del complejo costó 480 millones de $€$, a los que se deben añadir los 25 millones de $€$ que, aproximadamente, costaron los terrenos considerados como rústicos en el momento de compra, a un precio medio de 2.500 ptas. $\mathrm{m}^{2}$ (15 $\left.€ . m^{2}\right)$ y que seguidamente fueron recalificados como suelo urbanizable. En la actualidad el precio medio del suelo urbano en Boadilla del Monte es de $346 €^{8}$ el metro cuadrado, por lo que la recalificación ya generó pingües beneficios.

¿Las compras corporativas masivas, tanto extranjeras como nacionales, de edificios urbanos y terrenos que despegaron después de la crisis de 2008 señalan una nueva fase emergente en las principales ciudades? (Sassen, 2015).

Las plusvalías derivadas de la recalificación de los terrenos y la posterior venta de todo el complejo al grupo inversor "Marme Inversiones", perteneciente al Grupo inmobiliario británico Propinvest, fueron muy elevadas. La operación de venta se cerró en el año 2008, aun en plena

8 Fuente: https://www.certicalia.com/precio/valoracion-suelo-urbano/madrid/madrid burbuja inmobiliaria, por 1.900 millones de $€$, lo que le supuso al banco unos beneficios brutos de 1.395 millones de $€$, además de aportarle liquidez. El contrato de compraventa también incluía el alquiler del complejo al propio banco por 82 millones de $€$ anuales durante 40 años, pero esa cifra estaba condicionada a la ocupación real de los edificios. Con la llegada de la crisis económica de 2008, y la incorporación jurídica de otras entidades bancarias como Banesto o Popular Español, el Banco Santander, para minimizar los altos costes de alquiler de la Ciudad Financiera, comenzó a trasladar a sus empleados a los inmuebles de los bancos adquiridos con lo que, al estar infrautilizado el recinto, descendía el precio de alquiler.

Dos años después de realizar la venta de la Ciudad Financiera a Marme Inversiones la entidad financiera volvió a adquirir $901.000 \mathrm{~m}^{2}$ de terrenos situados junto al campo de golf de la propia ciudad, terrenos que fueron subastados por el Ministerio de Defensa, para lo cual también tuvo que abonar al Ayuntamiento de Boadilla del Monte 2,1 millones de $€$ a cambio de las cesiones de derechos que pertenecían al Ayuntamiento. Una gran parte de estos terrenos $\left(711.579 \mathrm{~m}^{2}\right)$ tienen la consideración de suelo urbanizable de especial protección. El resto se trata de suelo urbanizable con una edificabilidad aproximada de 40.000 metros.

Todo este proceso refleja una nueva realidad de utilización del suelo y que se reproduce en otras muchas ciudades del mundo, donde las inversiones inmobiliarias de las grandes corporaciones bancarias y fondos financieros se 
incrementan. Hoy en día hay alrededor de 100 ciudades en todo el mundo que han emergido como destinos importantes para tales adquisiciones: las compras corporativas extranjeras de propiedades de 2013 a 2014 crecieron un 248\% en Ámsterdam / Randstad, un 180\% en Madrid y un 475\% en Nanjing (Sassen, 2015).

No se ha podido averiguar a qué va a destinar el Banco Santander tales terrenos, si bien, al tratarse de suelo urbanizable es posible que, a medio o largo plazo, sea enajenado en búsqueda de plusvalías; no obstante, este nuevo modelo inversor es posible que no represente una tendencia perdurable en el tiempo, sino que principalmente tiene su origen en el actual modelo financiero internacional en el que la rentabilidad de la denominada renta fija es muy baja, secuela de lo barato del precio del dinero. En esta situación monetaria internacional los bancos buscan otros tipos de negocio en los que el retorno de las inversiones realizadas sea superior a los beneficios derivados de la tradicional actividad bancaria de depósitos y préstamos.

En el presente la propiedad de la Ciudad Financiera de Boadilla del Monte está en litigio después de que Marme Inversiones presentase Suspensión de Pagos y todo el conjunto saliese a subasta pública ${ }^{9}$.

El efecto más inmediato de las compras corporativas masivas de inmuebles y suelo urbano por parte de los grandes inversores (bancos y fondos), tanto nacionales como internacionales, es la elevación artificial del precio de los bienes inmuebles ur-

9 Marme Inversiones, ante la imposibilidad de pagar el crédito con el que financió la compra de la Ciudad Financiera, esta salió a pública subasta, habiéndose interesado por su adquisición diferentes fondos de inversión extranjeros como Blackstone o el propio Banco Santander entre otros. Finalmente, la adjudicación, aunque litigada por el Banco Santander, ha ido a parar a manos del fondo capital de inversión: Sorlinda Investments SL, vinculado a un conglomerado inglés perteneciente a los hermanos Simon y David Reuben. El fuerte interés desatado por la subasta pública, junto al hecho de que el banco haya recurrido su adjudicación y advertido que, en caso de fallo judicial negativo, se reserva ejercer el derecho de tanteo que tiene por ley, al ocupar actualmente estos edificios, ratifica la idea arriba mencionada de que los inmuebles y terrenos en determinadas ciudades y áreas metropolitanas se consideran como medios especulativos en busca de plusvalías. banos, la incorporación de nuevos modelos constructivos y rehabilitadores, ajenos a las necesidades de la población residente en la ciudad, al convertir los inmuebles y el suelo urbano en un producto financiero más de detracción de plusvalías.

\section{CONCLUSIONES}

Los nuevos procesos de localización espacial empresarial que están surgiendo en Madrid y en su corona metropolitana, ligados al sector financiero, no pueden ser analizados exclusivamente en clave de conectividad, economía de aglomeración, renta de situación. Las inversiones en suelo, al haber descendido los márgenes procedentes del tradicional modelo productivo bancario (préstamos e hipotecas), tanto urbano como rural, se han convertido en un producto financiero de obtención de ganancias derivadas de las plusvalías, tanto para bancos como para fondos de inversión y grupos inmobiliarios. Este proceso refleja una nueva realidad de utilización del suelo, no solo en Madrid sino en muchas ciudades del mundo, sobre todo en aquellas más importantes, donde las inversiones inmobiliarias de las grandes corporaciones bancarias y fondos financieros se incrementan en búsqueda de ganancias. Madrid no ha quedado al margen de este proceso.

En la actualidad, y como consecuencia de las diferentes operaciones de integraciones financieras y de adquisiciones bancarias que comenzaron ya en el último cuarto del siglo XX y continuaron con la crisis económica del año 2008, crisis protagonizada en España principalmente por las denominadas Cajas de Ahorro, pocos bancos mantienen sus sedes sociales en la capital de la nación. Los dos grandes bancos internacionales, Santander y BBVA, en Madrid tan solo tienen sus sedes corporativas y operativas, y estas han sido deslocalizadas a distritos de borde o espacios metropolitanos, actuaciones que deben ser entendidas como un medio para conseguir plusvalías al desprenderse de alguno de sus edificios en aquellos espacios de mayor centralidad.

El caso que nos ocupa del Banco Santander y la deslocalización de su sede corporativa hacia Boadilla del Monte, le sirvió al banco para incre- 
mentar sus beneficios derivados de las grandes plusvalías obtenidas de la recalificación de su suelo de rural a terciario y para conseguir liquidez.

En la actualidad la Ciudad Financiera, cuya propiedad está recurrida en los tribunales, ha perdido parte de sus trabajadores, que han sido trasladados a las oficinas del recién absorbido Banco Popular Español. Queda por determinar el destino final del espacio si los tribunales fallan a favor del Banco Santander respecto a la propiedad de dichos terrenos. En cualquier caso, la relocalización de empleados desde espacios de gran valor en el interior urbano madrileño, como

\section{REFERENCIAS}

Ardua Urquiaga, A. (2014). De Chirico en BoadiIla. La ciudad financiera del BSCH (pp. 221248). En J. Echenagusia (Coord.) Madrid: materia de debate. Madrid: Club de Debates urbanos.

Arnaiz Consultores S.L. (2007). Boadilla del Monte un lugar para vivir. Madrid: Grupo Códice.

Ayuntamiento de Boadilla del Monte (2003). Boadilla del Monte. Modelo de futuro. Madrid: Arnaiz Consultores.

Ayuntamiento de Boadilla del Monte (2017). Nuevo Plan de Ordenación Urbana de Boadilla del Monte. Madrid. Recuperado de https://ayuntamientoboadilladelmonte.org/sites/default/files/vol_1_ doc_i._01-memoria_y_anexos_1_y_2_0.pdf.

Beaujeu-Garnier, J y Chabot, G. (1963). Traité de Géographie Urbaine. Paris: Colin.

Época (2003). Boadilla del Monte. Pulmón de calidad. Grupo Intereconomía, 950, del 2 al 8 de mayo, 71-78.

Feria Toribio, J.M. (2010). La movilidad residencial y los procesos de urbanización metropolitanos en España (pp. 23-47). En J. Feria Toribio y J. Albertos Puebla (Coord) La Ciudad Metropolitana en España: procesos urbanos en los inicios del siglo XXI. Navarra: Civitas (Thomson Reuters).

George, P. (1961). Précis de géographie urbaine. Paris: Presses Universitaires de France.

Herbert, D.T. y Thomas, C.J. (1990). Cities in space: cities as place. London: Routledge.

Instituto de estadística de la Comunidad de Ma- los que trabajaban en el denominado complejo Banesto-Mesena, hacia distritos periféricos de menor renta de situación sigue siendo un medio de conseguir mejorar la cuenta de resultados e incrementar la liquidez de las empresas.

Que el suelo se haya convertido en un producto financiero más a escala mundial introduce en los procesos urbanos grandes disrupciones en el uso tradicional del suelo urbano, generando importantes tensiones entre la población residente, que ve como se incrementa artificialmente el precio de éste, y los inversores en busca de elevados beneficios.

drid (2016). Padrón continuo 2016. Municipios de la Comunidad de Madrid, distritos y barrios de Madrid y zonas estadísticas.

Jodidio, P. (2012). Ciudad Grupo Santander: arquitectura \& naturaleza. Madrid: Grupo Santander.

Meuriot-P (2018). Des Agglomerations Urbaines

Dans L'Europe Contemporaine: Essai Sur Les Causes. París. Ed. Hachette Livre-BNF.

Martín Roda, E.M. (2000). Madrid eje terciario. Madrid: revista de arte, geografía e historia, 3, 231-255. Comunidad de Madrid: Consejería de Educación. Mazo, E. (2016). Forbes coloca a 26 empresas españolas entre las 2.000 más grandes del mundo. Expansión.com. Recuperado de http:// www.expansion.com/empresas/2016/05/26/ 5746bb2cca4741e37a8b4650.html

Perelló Ferrer, M. A. (1991). Las claves de la arquitectura. Madrid: Planeta.

Pérez Mejías, C. (2012). Características de los distritos financieros en el espacio urbano. GeoGraphos. Revista Digital para Estudiantes de Geografía y Ciencias Sociales, 3(22). Recuperado de https://web.ua.es/es/revista-geographos-giecryal/documentos/distritos-financieros.pdf

Rodríguez-Rata, A. (26 de marzo 2018). Saskia Sassen: "No es el 1\%; un $30-40 \%$ es súper rico, el resto se empobrece". Ciudad-super-ricos-pobres. La Vanguardia. Recuperado de https://www.lavanguardia.com/ economia/20180326/441862316018/conver- 
saciones-saskia-sassen-ciudad-super-ricos-pobres.html

Saldarriaga Roa, A. (2016). Acerca de las ciudades: la mirada de ayer y de hoy. Procesos urbanos, 3, 10-23.

Salom, J. y Albertos Puebla, J.M. (2010). Densidad de la red viaria y forma urbana: delimitación del espacio urbano en ocho aglomeraciones españolas (pp.49-94). En J. Feria Toribio y J. Albertos Puebla (Coord.) La Ciudad Metropolitana en España: procesos urbanos en los inicios del siglo XXI. Navarra: Civitas (Thomson Reuters).

Sánchez Moral, S; Calatrava Andrés, A. y Melero Guilló, A. (2008). Las funciones comando de Madrid en la economía global: una aproximación a través del proceso de atracción de capital extranjero. Revista Eure, XXXIV(101), 25-44. Sanz García, J.M. (1975). Madrid ¿Capital del capital español? Madrid: Instituto de Estudios Madrileños.

Sassen, S. (2007). El reposicionamiento de las ciudades y regiones urbanas en una economía global: ampliando las opciones de políticas y gobernanza. Revista Eure, XXXIII(100), 9-34. Recuperado de https://scielo.conicyt.cl/pdf/ eure/v33n100/art02.pdf

Sassen, S. (2015). Who owns our cities - and why this urban takeover should concern us all. The Guardian.
Recuperado de https://www.theguardian.com/ cities/2015/nov/24/who-owns-our-cities-andwhy-this-urban-takeover-should-concern-us-all Solís Trapero, E. (2011). Del área metropolitana hacia la región urbana policéntrica madrileña: cambio de escala, estructura y articulación territorial. Tesis doctoral. Universidad Complutense.

Sudjic, D. (2009). La arquitectura del poder. Barcelona: Ariel.

Tobío Soler, C. (1987). La reestructuración económico-social en el área metropolitana de Madrid 19701985. Tesis doctoral. Universidad Complutense. Torquemada, B. (2012). Bienvenidos a la ciudad de Botín. ABC. Recuperado de https://www.abc. es/20120508/sociedad/abci-bienvenidos-ciudad-botin-santander-201205071935.html

\section{OTRAS FUENTES}

www.santander.es

h t t p s: / / sext a columna.wordpress. com/2012/11/12/relato-de-la-fiesta-ii/

https://www.certicalia.com/precio/valoracion-suelo-urbano/madrid/madrid/madrid

https://www.idealista.com/ . Revista de compra venta de inmuebles

Eva María Martín Roda es Doctora en Geografía por la UCM-1999. Profesora a tiempo completo en la UNED. Ha formado parte como investigadora de diversos proyectos de ámbito nacional y europeo. Como IP ha dirigido los proyectos titulados: El Canal de Castilla: la infrautilización de un patrimonio industrial del siglo $X I X$ y ha sido IP de la parte técnica del proyecto titulado: La empresa multinacional en la economía madrileña, realizado por el IEE. Es Miembro del grupo de Investigación Geografía, Paisaje y TIC. Ha publicado diferentes libros y artículos en revistas como: BAGE; Didáctica Geográfica; Economistas; Anales de Geografía de la UCM; BBV-Situación o Espacio, Tiempo y Forma; aparece en diferentes catálogos como: BNE, ISNI, Library of Congress y VIAF. Es miembro del Consejo de Redacción de la revista Espacio Tiempo y forma. Serie VI-Geografía y colabora como revisora en la revista Tourism Management. Departamento de Geogafía. Facultad de Geografía e Historia. Universidad Nacional de Educación a Distancia (UNED). Paseo Senda del Rey, 7. Edificio de Humanidades, $4^{\circ}$ y $5^{\circ}$ planta, (28040) Madrid, España, emartin@geo.uned.es

Silvana Sassano Luiz es Doctora en Geografía por la Universidad Complutense de Madrid-2016. Profesora-Tutora de la Universidad Nacional de Educación a Distancia (UNED). Ha sido Ayudante de investigación en el Instituto de Geografía de la Universidad de Buenos Aires; ha trabajado en el Ministerio de Educación de la República Argentina en la Secretaría de Políticas Universitarias en el Programa "Mejoramiento de la calidad universitaria". Profesora e investigadora en la Universidad General Sarmiento. Profesora en diversos Institutos de Educación Secundaria. Investigadora en la Universidad Complutense de Madrid en diversos proyectos sobre "Población inmigrante extranjera en la Comunidad de Madrid", "Consumo y Ciudad en la Comunidad de Madrid". Ha publicado libros y artículos sobre Geografía del consumo, migraciones, innovación educativa, urbanismo. Fue miembro del Programa "Redes para la innovación docente" Departamento de Geogafía. 